\title{
APPROXIMATE SOLUTION OF SECOND KIND INTEGRAL EQUATIONS ON INFINITE CYLINDRICAL SURFACES
}
A.T. Peplow and S.N. Chandler-Wilde 
w9253524 


\section{APPROXIMATE SOLUTION OF SECOND KIND INTEGRAL EQUATIONS ON INFINITE CYLINDRICAL SURFACES}

\section{ANDREW T. PEPLOW* AND SIMON N. CHANDLER-WILDE ${ }^{\uparrow}$}

Abstract. The paper considers second kind integral equations of the form $\phi(x)=g(x)+$ $\int_{S} k(x, y) \phi(y) d s(y)$ (abbreviated $\left.\phi=g+K \phi\right)$, in which $S$ is an infinite cylindrical surface of arbitrary smooth cross-section. The "truncated equation" (abbreviated $\phi_{a}=E_{a g}+K_{a} \phi_{a}$ ), obtained by replacing $S$ by $S_{a}$, a closed bounded surface of class $C^{2}$, the boundary of a section of the interior of $S$ of length $2_{a}$, is also discussed. Conditions on $k$ are obtained (in particular, implying that $K$ commutes with the operation of translation in the direction of the cylinder axis) which ensure that $I-K$ is invertible, that $I-K_{a}$ is invertible and $\left(I-K_{a}\right)^{-1}$ uniformly bounded for all sufficiently large $a$, and that $\phi_{a}$ converges to $\phi$ in an appropriate sense as $a \rightarrow \infty$. Uniform stability and convergence results for a piecewise constant boundary element collocation method for the truncated equations are also obtained.

A boundary integral equation, which models three-dimensional acoustic scattering from an infinite rigid cylinder, illustrates the application of the above results to prove existence of solution (of the integral equation and the corresponding boundary value problem) and convergence of a particular collocation method.

Key words. second kind integral equations, Wiener-Hopf equations, boundary element method, Helmholtz equation, collocation method

AMS subject classifications. 65R20, 45E10, 65N38, 35J05

1. Introduction. We are concerned in this paper with second kind integral equations of the form

$$
\phi(x)=g(x)+\int_{S} k(x, y) \phi(y) d s(y), \quad x \in S,
$$

and their numerical solution, in the case when $S$ is an infinite cylindrical surface with arbitrary cross-section in three-dimensional space. In equation (1) $g \in B C(S)$ (the space of bounded continuous functions on $S$ ) is assumed known and $\phi \in B C(S)$ is to be determined. We abbreviate (1) in operator form as

$$
\phi=g+K \phi
$$

and make sufficient assumptions on the smoothness of the surface $S$ and on the behaviour of the kernel $k$ ( $k$ is continuous or weakly singular) so that $K: B C(S) \rightarrow$ $B C(S)$ is bounded but not compact. In particular, we suppose that

$$
S=\left\{\left(x_{1}, x_{2}, x_{3}\right):\left(x_{1}, x_{2}\right) \in \Gamma, x_{3} \in \mathrm{R}\right\}
$$

where $\Gamma \subset \mathrm{R}^{2}$ is a Jordan curve of class $C^{2}$.

Integral equations of the form (1) frequently arise when reformulating linear elliptic boundary value problems in the interior or exterior of $S$ as boundary integral equations. We consider an example of this type in Section 4 of the paper, in which acoustic scattering by an infinite rigid cylinder is investigated, with

$$
k(x, y)=2 \frac{\partial}{\partial n(y)} G(x, y),
$$

* Structural Dynamics Group, Institute of Sound and Vibration Research, University of Southampton, Southampton S09 5NH, UK (A.T.Peplow@isvr.soton.ac.uk).

$\dagger$ Department of Mathematics and Statistics, Brunel University, Uxbridge UB8 3PH, UK (Simon.Chandler-Wilde@brunel.ac.uk) 
where $n(y)$ is the normal to $S$ at $y$, directed into the exterior of $S$, and

$$
G(x, y):=\frac{e^{i \kappa|x-y|}}{4 \pi|x-y|}
$$

is a fundamental solution of the Helmholtz equation, $\Delta u+\kappa^{2} u=0$.

When solving (1) numerically it is convenient, as a preliminary stage, to truncate the infinite surface $S$. Let $\widetilde{S}_{+}$and $\widetilde{S}_{-}$denote the half cylinders denned by

$$
\widetilde{S}_{ \pm}:=\left\{\left(x_{1}, x_{2}, x_{3}\right) \in S: x_{3} \gtrless 0\right\}
$$

and, for $a \geq 2$, let

$$
\widetilde{S}_{a}:=\left\{\left(x_{1}, x_{2}, x_{3}\right) \in S:\left|x_{3}\right| \leq a\right\} .
$$

Let $E_{-}$be the surface

$$
E_{-}:=\left\{\left(x_{1}, x_{2}, x_{3}\right):\left(x_{1}, x_{2}\right) \in \bar{\Omega}, x_{3}=f\left(x_{1}, x_{2}\right)\right\}
$$

where $\Omega$ is the interior of $\Gamma$, and $f$ is any given continuous function on $\bar{\Omega}$ satisfying

$$
\begin{array}{ll}
f\left(x_{1}, x_{2}\right) & >0, \quad\left(x_{1}, x_{2}\right) \in \Omega, \\
=0, & \left(x_{1}, x_{2}\right) \in \Gamma,
\end{array}
$$

and such that $S_{-}:=\widetilde{S}_{-} \cup E_{-}$is a smooth surface of class $C^{2}$ (see Figure 1). Let $E_{+}:=\left\{\left(x_{1}, x_{2}, x_{3}\right):\left(x_{1}, x_{2}\right) \in \bar{\Omega}, \quad x_{3}=f\left(x_{1}, x_{2}\right)\right\}$ and $S_{+}:=\widetilde{S}_{+} \cup E_{+}$.

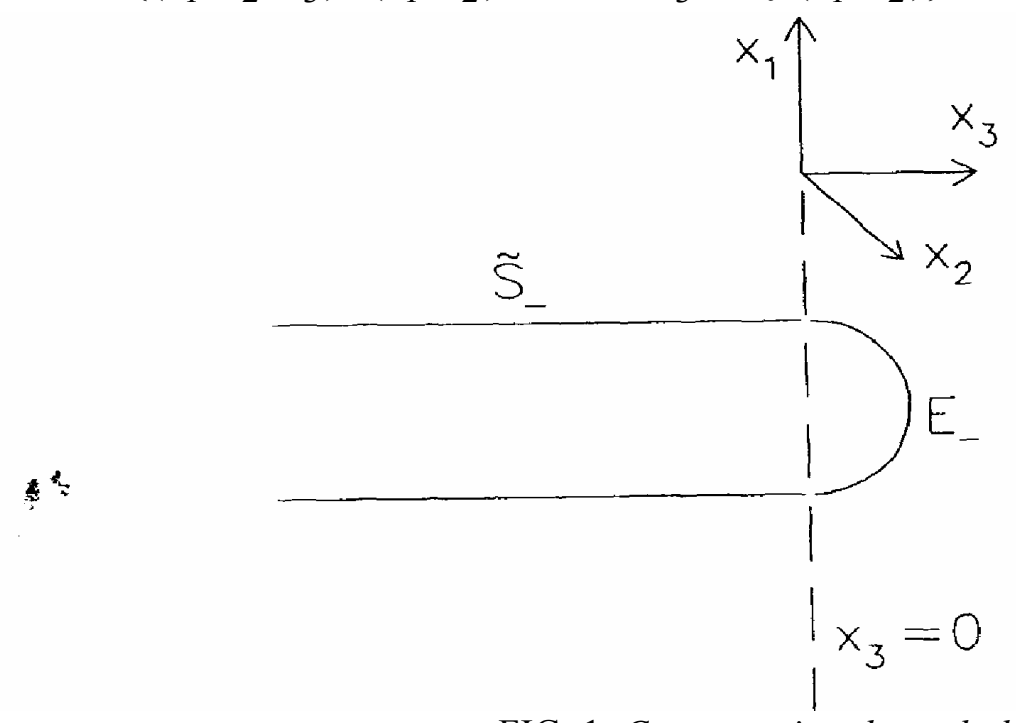

FIG. 1. Cross-section through the surface $S_{-}$.

For $V \subset \mathrm{R}^{3}$ and $x \in \mathrm{R}^{3}$, let $V+x$ denote the translation of the set $V$ by the vector $x$, and let $e_{3} \in \mathrm{R}^{3}$ be the unit vector in the $x_{3}$ direction. Then, for $a \geq 2$, define $S_{a}:=\quad \widetilde{S}_{a} \cup\left(E_{-}+a e_{3}\right) \cup\left(E_{+}-a e_{3}\right)=\widetilde{S}_{a} \cup\left\{\left(x_{1}, x_{2} x_{3}\right):\left(x_{1}, x_{2}\right) \in \bar{\Omega}, x_{3}=\right.$ $\left.\pm\left(a+\int\left(x_{1}, x_{2}\right)\right)\right\}$ (see Figure 2). Note that $S_{a}$ is a smooth closed surface of class $C^{2}$.

Let $\Sigma:=\left\{S, S_{+}, S_{-}\right\} \cup\left\{S_{a}+b e_{3}: a \geq 2, b \in \mathrm{R}\right\}$ and, for $T \subset S^{*} \in \sum$, define the integral operator $K_{T}$ on $B C(T)$ by

$$
K_{T} \psi(x)=\int_{T} k(x, y) \psi(y) d s(y) \quad x \in T .
$$




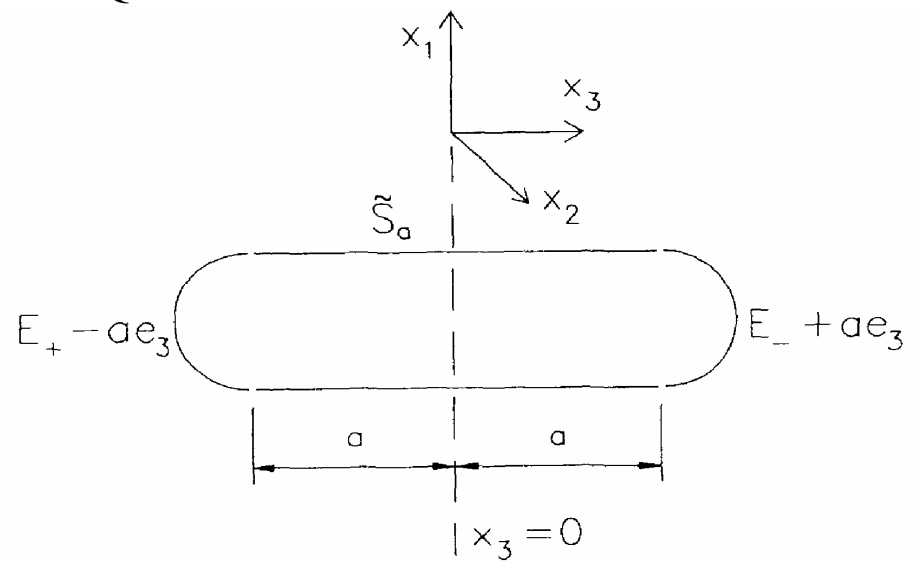

FIG. 2. Cross-section through the surface $S_{a}$.

Let $K \pm: K_{S \pm}$ and $K_{a}:=K_{S_{a}}$, for $a \geq 2$.

A crucial requirement for the theory developed is a translation invariant assumption on the kernel function $k$, that, for all $(x, y) \in I:=\{(x, y) \in T: T \in \Sigma\}$,

$$
k\left(x+t e_{3}, y+t e_{3}\right)=k(x, y), \quad t \in \mathrm{R} .
$$

We analyse, in Section 2 of this paper, the approximation to (1) obtained by replacing the infinite surface $S$ by the finite closed surface $S_{a}$ and the convergence to $\phi$, of the solution $\phi_{a} \in B C\left(S_{a}\right)$ of the approximate equation, as $a \rightarrow \infty$. To make precise the definition of $\phi_{a}$ and the sense in which $\phi_{a} \in B C\left(S_{a}\right)$ approximates $\phi \in B C(S)$, introduce mappings between these two spaces. For $a \geq 1$, let $F_{a} \in C^{\infty}(\mathrm{R})$ be an even function satisfying $0 \leq F_{a}(t) \leq 1, t \in \mathrm{R}$, , and

$$
F_{a}(t)=\quad \begin{aligned}
& 1, \quad 0 \leq t \leq a \\
& 0, \quad t \geq a+1
\end{aligned}
$$

For $a \geq 2$ define $E_{a}: B C(S) \rightarrow B C\left(S_{a}\right)$ by

$$
E_{a} \psi(x):= \begin{cases}F_{a-1}\left(x_{3}\right) \psi(x), & x=\left(x_{1}, x_{2}, x_{3}\right) \in \tilde{S}_{a}, \\ 0, & x \in S_{a} \backslash \tilde{S}_{a},\end{cases}
$$

and $R_{a}: B C\left(S_{a}\right) \rightarrow B C(S)$ by

(8) $R_{a} \psi(x):=\quad \begin{cases}\psi(x), & x \in \tilde{S}_{a}, \\ \psi\left(\left(x_{1}, x_{2}, a\right)\right) F_{a}\left(x_{3}\right), & x=\left(x_{1}, x_{2}, x_{3}\right) \in S \backslash \tilde{S}_{a}, \quad x_{3} \geq a, \\ \psi\left(\left(x_{1}, x_{2},-a\right)\right) F_{a}\left(x_{3}\right), & x=\left(x_{1}, x_{2}, x_{3}\right) \in S \backslash \tilde{S}_{a}, \quad x_{3} \leq-a .\end{cases}$

Then $\phi_{a} \in B C\left(S_{a}\right)$ is defined by

$$
\phi_{a}=E_{a} g+K_{a} \phi_{a},
$$

which we will refer to as the "truncated" version of (1).

In Section 2 we construct a partial theory of the solvability of equation (1) and of the truncated equation (9). Theorem 2.8 suggests that the existence of a solution 
to the truncated equation for all sufficiently large $a$ depends not just on the unique solvability of the original integral equation (1) but also on that of the "half cylinder" equations obtained by replacing $S$ by $S \pm$. Specifically, it shows that $I-K_{a}$ is invertible and uniformly bounded for all sufficiently large a provided $I-K, I-K_{+}$and $I-K_{-}$ are injective. These conditions also ensure the invertibility of the original operator $I-K$ (Corollary 2.9), so that the spectrum of $K$ is contained in the union of $\{0\}$ and the sets of eigenvalues of $K, K_{+}$, and $K$. This result may be powerful for establishing existence of a solution to equation (1) and, in the case when (1) is a boundary integral equation, for establishing existence of solution for the corresponding boundary value problem formulation. These points are illustrated by the example in Section 4.

In Section 3 we consider the numerical solution of the integral equation on $S_{a}$, defining $\phi^{(n)} \in B C\left(S_{a_{n}}\right)$ by

$$
\phi^{(n)}=E_{a_{n}} g+K^{(n)} \phi^{(n)},
$$

where $K^{(n)}=K_{a_{n}} P_{n}$ and $P_{n}$ is an interpolatory projection operator onto a space of piecewise constant functions on a finite element mesh on $S_{a_{n}}$ Note that $P_{n} \phi^{(n)}$ is a piecewise constant collocation method approximation to $\phi_{a_{n}}$. and $\phi^{(n)}$ is the iterated collocation method approximation of Sloan [12]. The results of Section 2 are extended to show that the operators $\left(I-K^{(n)}\right)^{-1}$ are uniformly bounded for all sufficiently large $n$ and that $R_{a_{n}} \phi^{(n)}$ converges to $\phi$ uniformly on compact subsets of $S$ provided that $a_{n} \rightarrow+\infty$ and $h_{n} \rightarrow 0$ as $n \rightarrow \infty$, where $h_{n}$ is the diameter of the largest element of the mesh on $S_{a_{n}}$. Further, if $\phi(x) \rightarrow 0$ as $x \rightarrow \infty, R_{a_{n}} \phi^{(n)}$ converges to $\phi$ uniformly on $\mathrm{S}$,

The integral equation (9) may seem a perverse choice as approximation for (1): the approximation obtained by replacing $S$ by $\widetilde{S}_{a}$ in (1) may seem more obvious: indeed, this alternative approximation can be analysed in a similar (in fact simpler) manner. However, the resulting theory appears to be inapplicable in practical situations in which (1) is a boundary integral equation. In such applications it is generally the case that (1) with $S$ replaced by $S_{ \pm}$is still a boundary integral equation so that the injectivity of $I-k_{ \pm}$can be established in a similar manner to that of $I-K$ (cf. Section 4). Equation (1) with $S$ replaced by $\widetilde{S}_{ \pm}$is generally not a boundary integral equation, and it is not clear, in practical cases, how the injectivity of $I k_{\tilde{S}_{ \pm}}$(which the alternative theory requires) might be established.

Integral equations on smooth closed bounded surfaces in $\mathrm{R}^{3}$ and their numerical treatment have a wide literature: see Colton and Kress $[9,10]$ for that part relevant to the acoustic scattering example of Section 4. The piecewise constant collocation method discussed in Section 3 is the most commonly used boundary element method (Brebbia et al. [6]). For integral equations of the class discussed in this paper on smooth bounded surfaces, the stability and convergence of this boundary element method can be analysed using standard results from the collectively compact operator theory of Anselone [1],

To the best of our knowledge, this paper is the first attempt to develop a theory for integral equations of the form (1) and their numerical solution. Our arguments generalise ones in collectively compact operator theory [1]. Our assumptions, results, and methods of proof are closest to those of Atkinson [5], Anselone and Sloan [2-4], and Chandler-Wilde [8], who consider the approximate solution of integral equations on the real line.

2. The Original and Truncated Equations. Let $k_{x}(y)=k(x, y),(x, y) \in I$. We suppose throughout that, for all $a \geq 2$ and $x \in S_{a}, k_{x} \in L_{1}\left(S_{a}\right)$, and that $k$ 
satisfies the following assumptions, A1-A4:

A1. For all $(x, y) \in I=\left\{(x, y) \in T: T \in \sum\right\}, \quad k\left(x+t e_{3}, y+t e_{3}\right)=k(x, y), \quad t \in \mathrm{R}$.

A2. $c:=\sup _{a \geq 2, x \in S_{a}} \int_{S_{a}}|k(x, y)| d s(y)<\infty$.

A3. $\Delta(h):=\sup _{a \geq 2, x, x^{\prime} \in S_{a},\left|x \quad x^{\prime}\right| \leq h} \int_{S_{a}}\left|k(x, y)-k\left(x^{\prime}, y\right)\right| d s(y) \rightarrow 0$ as $h \rightarrow 0$.

A4. For $x=\left(x_{1}, x_{2}, x_{3}\right) \in S_{ \pm}, \int_{E_{ \pm}}|k(x, y)| d s(y) \rightarrow 0 \quad$ as $\quad x_{3} \rightarrow \pm \infty$.

Let $B_{T}:=\left\{\psi \in L_{\infty}(T):\|\psi\|_{\infty} \leq 1\right\}$, for all $T \in \sum$. Note that Assumptions A1-A3 imply that

$\bigcup K_{T} B_{T}$ bounded and equicontinuous,

in that

and

$$
\sup _{T \in \sum, \psi \in B_{T}}\left\|K_{T} \psi\right\|_{\infty} \leq C
$$

$$
\sup _{T \in \Sigma, \psi \in B_{T}, x, x^{\prime} \in T,\left|x \quad x^{\prime}\right| \leq h}\left|K_{T} \psi\left(x^{\prime}\right)\right| \geq \Delta(h) .
$$

Thus, if Assumptions A1-A3 are satisfied, then $K_{T}$ is a bounded operator from $L \infty(T)$ to $B C(T)$ for each $T \in \sum$ (indeed, $K_{T}$ is compact if $T$ is bounded), and

$$
\sup _{T \in \sum}\left\|K_{T}\right\| \leq c
$$

From A1-A3 we have also the following technical lemma:

LeMma 2.1. Define $\Phi_{+}(t), \Phi_{-}(t)$, for $t \geq 0$, by

$$
\Phi_{ \pm}(t):=\sup _{x \in \widetilde{\Gamma} \mp t e 3} \int_{\widetilde{S}_{ \pm}}|k(x, y)| d s(y),
$$

Where $\widetilde{\Gamma}:=\left\{\left(x_{1}, x_{2}, 0\right):\left(x_{1}, x_{2}\right) \in \Gamma\right\}$, and $\Phi(t)$ by $\Phi(t):=\max \left(\Phi_{+}(t), \Phi_{-}(t)\right)$. Then $\Phi(t) \rightarrow 0$ as $t \rightarrow+\infty$.

Proof. Consider $\Phi_{+}(\mathrm{t})$. From Assumption A2, $\sup _{x \in S} \int_{S}|k(x, y)| d s(y)<\infty$, so that $\Phi_{+}$is well denned and

$$
\int_{\widetilde{S}_{+}+t e_{3}}|k(x, y)| d s(y) \rightarrow 0
$$

as $t \rightarrow+\infty$ with $x$ fixed. Applying Al, it follows that

$$
\int_{\widetilde{S}_{+}}|k(x, y)| d s(y) \rightarrow 0
$$

as $t \rightarrow+\infty$ with $x=\left(x_{1}, x_{2},-t\right)$ and $\left(x_{1}, x_{2}\right) \in \Gamma$ fixed. But, by Assumption A3 $\int_{\widetilde{S}_{+}}|k(x, y)| d s(y)$ uniformly continuous on $S$. It follows that the convergence (13) 
is uniform in $\left(x_{1}, x_{2}\right) \in \Gamma$, so that $\Phi_{+}(t) \rightarrow 0$ as $t \rightarrow+\infty$. Similarly, $\Phi_{-}(t) \rightarrow 0$ as $t \rightarrow+\infty$.

Assumption A4 gives rise to a similar result concerning the integral over $E_{ \pm}$.

LEMMA 2.2. Define $\Psi_{+}(t), \Psi(t)$, for $t \geq 0$, by

$$
\Psi_{ \pm}(t):=\sup _{x \in \widetilde{\Gamma} \pm t e_{3}} \int_{E_{ \pm}}|k(x, y)| d s(y),
$$

and $\Psi(t)$ by $\Psi(t):=\max \left(\Psi_{+}(t), \Psi_{-}(t)\right)$. Then $\Psi(t) \rightarrow 0$ as $t \rightarrow+\infty$.

Proof. From Assumption A2, $\Psi$ is well defined and, from Assumptions A2 and A3, $\int_{E_{ \pm}}|k(x, y)| d s(y)$ is uniformly continuous on $S_{ \pm}$. It follows that $\Psi(\mathrm{t}) \rightarrow 0$ from Assumption A4.

We shall employ principally the following notions of convergence. For a sequence $\left\{T_{n}\right\}$ $\subset \sum$ and $T \in \sum$ we shall write $T_{n} \rightarrow T$ if, for all $A>0,\left\{x \in T_{n}:|x| \leq A\right\}=\{x \in T:|x| \leq A\}$ for all sufficiently large $n$. For a sequence $\left\{\psi_{n}\right\}$, with $\psi_{\mathrm{n}} \in L_{\infty}\left(T_{n}\right)$, we shall write $\psi_{n} \rightarrow \psi \in L_{\infty}(T)$ if $T_{n} \rightarrow T, \sup _{n}\left\|\psi_{n}\right\|_{\infty}<\infty$, and, for all $A>0$, ess $\sup _{x \in T,|x| \leq A}\left|\psi_{n}(x)-\psi(x)\right| \rightarrow 0$ as $n \rightarrow \infty$.

REMARK 2.1. If $T_{n} \rightarrow T$ then, for all sufficiently large $n, T_{n} \backslash T \subset\left(E_{+}-b_{n}\right) \cup$ $\left(E_{-}+c_{n}\right)$, where $b_{n}, c_{n} \geq 0$ and $b_{n}, c_{n} \rightarrow+\infty$ as $n \rightarrow \infty$. Thus, if $T$ is bounded, $T_{n}=T$ for all sufficiently large $n$, so that, if $\psi \in L_{\infty}(T)$, then $\psi_{n} \rightarrow \psi \Leftrightarrow\left\|\psi_{n}-\psi\right\|_{\infty} \rightarrow 0$.

REMARK 2.2. If $\psi_{n} \in B C\left(T_{n}\right)$ for each $n$ then $\psi \in B C(T)$.

REMARK 2.3. If $\psi_{\mathrm{n}} \rightarrow \psi$ then $\|\psi\|_{\infty} \leq \sup _{\mathrm{n}}\left\|\psi_{n}\right\|_{\infty}$.

REMARK 2.4. If $\psi_{n} \in B C(T)$ and $\psi_{n} \in B C(T)$. for each $n$, then the convergence $\psi_{n} \rightarrow \psi$ is strict convergence in the sense of Buck [7].

REMARK 2.5. A useful test of convergence is: $\psi_{n} \rightarrow \psi \Leftrightarrow$ every subsequence of $\left\{\psi_{n}\right\}$ has a subsequence that converges to $\psi$.

Our next two results are, respectively, a collective compactness and a convergence property of the operator sequence $\left\{K_{T_{n}}\right\}$, in the case $T_{n} \rightarrow T$.

LEMMA 2.3. Suppose that $\left\{T_{n}\right\} \subset \Sigma, T_{n} \quad T \in \Sigma, \psi_{n} \in L_{\infty}\left(T_{n}\right)$ for each $n$, and $\sup _{\mathrm{n}}\left\|\psi_{\mathrm{n}}\right\|_{\infty}<\infty$. Then, for some subsequence $\left\{\psi_{n_{m}}\right\}, K_{T_{n_{m}}} \psi_{n_{m}} \rightarrow \psi \in B C(T)$.

Proof. Define $T_{A}^{*}:=\left\{\left(y_{1}, y_{2}, y_{3}\right) \in T ;\left|y_{3}\right| \leq A\right\}$, for $A>0$, and let $x_{n}=K_{T_{n}} \psi{ }_{n}$. Note that, for all $A>0, T_{A}^{*} \subset T_{n}$ for all sufficiently large $n$.

By (11), $\left\{x_{n}\right\}$ is bounded and equicontinuous. By the Arzela-Ascoli theorem and the above remarks, it follows that $\left\{x_{n}\right\}$ has a subsequence, $\left\{x_{n}^{(1)}\right\}$ which is a Cauchy sequence on $T_{1}^{*}$. Similarly, $\left\{x_{n}^{(m 1)}\right\}$ has a subsequence, $\left\{x_{n}^{(2)}\right\}$, which is a Cauchy sequence on $T_{2}^{*}$ Continuing the argument, we may construct, for each $m \in \mathrm{N}$, a subsequence $\left\{x_{n}^{(m)}\right\}$ of $\left\{x_{n}^{(m-1)}\right\}$ which is a Cauchy sequence on $T_{m}^{*}$. Then $\left\{x_{n}^{(n)}\right\}$ is a Cauchy sequence on $T_{m}^{*}$ for each $m \in \mathrm{N}$ and thus converges to an element of $B C(T)$.

LEMMA 2.4. Suppose that $\left\{T_{n}\right\} \subset \Sigma, T \in \Sigma, \psi_{n} \in L_{\infty}\left(T_{n}\right)$ for each $\mathrm{n}$, $\psi \in \mathrm{L}_{\infty}(\mathrm{T})$, and $\psi_{\mathrm{n}} \rightarrow \psi$. Then $K_{T_{n}} \psi_{n} \rightarrow K_{T} \psi$.

Proof. If $T$ is bounded then, by Remark 2.1, the above is no more than a statement that $K_{T}$ is continuous. Suppose that $T$ is unbounded. Since, by (11), $\left\{K_{T_{n}} \psi_{n}\right\}$ is bounded and equicontinuous, to show $K_{T_{n}} \psi_{n} \rightarrow K_{T} \psi$ we need only show pointwise convergence of $K_{T_{n}} \psi_{n}$ to $K_{T} \psi$

Let $\mathrm{C}:=\sup _{n}\left\|\psi_{\mathrm{n}}\right\|_{m}$, choose $x=\left(x_{1}, x_{2}, x_{3},\right) \in T$, and define $T_{A}^{*}$ as in the previous proof. For all $A>\left|x_{3}\right|$ and provided $T_{A}^{*} \subset T_{n}$ (true for all sufficiently large 
$n)$

$$
\begin{gathered}
\left|K_{T_{n}} \psi_{n}(x) \quad K_{T} \psi_{n}(x)\right| \leq\left|\int_{T_{A}^{*}}^{*} k(x, y)\left(\psi_{n}(y)\right) \quad \psi_{n}(y) d s(y)\right| \\
+C \int_{T_{n} \backslash T_{A}^{*}}|k(x, y)| d s(y)+C \int_{T \backslash T_{A}^{*}}|k(x, y)| d s(y) \\
\leq c \sup _{\left|y_{3}\right| \leq A}\left|\psi_{n}(y) \quad \psi(y)\right|+C \int_{\left(E_{+} b_{n}\right) \cup\left(E_{-}+C_{n}\right)}|k(x, y)| d s(y) \\
+2 C \int_{T \backslash T_{A}^{*}}|(x, y)| d s(y)
\end{gathered}
$$

for all sufficiently large $n$, where $b_{n}, c_{n} \geq 0$ and $b_{n}, c_{n} \rightarrow+\infty$ (To obtain this last inequality we have used Assumption A2, that, $T_{n} \backslash T_{A}^{*} \subset\left(T_{n} \backslash T\right) \cup\left(T \backslash T_{A}^{*}\right)$, and that, by Remark $2,1, T_{n} \backslash T C\left(E_{+}-b_{n}\right) \cup\left(E_{-}+c_{n}\right)$ for all sufficiently large $n$.) Applying Assumption Al and recalling the definitions of $\Psi$ and $\Phi$ we obtain

$$
\begin{aligned}
& \left|K_{T_{n}} \psi_{n}(\mathrm{x}) \quad K_{T} \psi(\mathrm{x})\right| \leq c \sup _{\left|y_{3}\right| \leq A}\left|\psi_{n}(\mathrm{y}) \quad \psi(y)\right|+C\left(\Psi\left(b_{n}+x_{3}\right)+\Psi\left(c_{n} \quad x_{3}\right)\right) \\
& +2 C\left(\Phi\left(A+x_{3}\right)+\Phi\left(A \quad x_{3}\right)\right) .
\end{aligned}
$$

Given $€>0$, by Lemma 2.1, the final term on the right hand side is $\leq € / 2$ if $A$ is chosen sufficiently large enough. Then, since $\psi_{\mathrm{n}} \rightarrow \psi$ and by Lemma 2.2, the remaining terms are $\leq \in / 2$ for all sufficiently large $n$. Thus $\left|K_{T_{n}} \psi_{n}(x) \quad K_{T} \psi_{n}(x)\right| \rightarrow 0$ as $n \rightarrow \infty$ for every fixed $x$.

We apply the above compactness and continuity properties first of all to give a condition for the continuous dependence of $\phi$ on $g$ in equation (1), in the case $g \in(I \quad K) B C(S)$.

THEOREM 2.5. If $I-K$ is injective then $(I-K)^{-1}$ exists and is a bounded operator on $(I-K) B C(S)$.

Proof. Suppose that the theorem is false. Then there exists a sequence $\left\{\psi_{\mathrm{n}}\right\} \subset$ $B C(S)$ with $\left\|\psi_{\mathrm{n}}\right\|_{\infty}=1$ for each $n$ such that $\left\|\psi_{n} K \psi_{n}\right\|_{\infty} \rightarrow 0$. Since $\left\|\psi_{n}\right\|_{\infty}=1$, we can find a sequence $\left\{a_{n}\right\} \subset \mathrm{R}$ such that $\sup _{x \in \widetilde{\Gamma}}\left\|\chi_{n}(x)\right\| \geq \frac{1}{2}$, where $\left\{\chi_{n}\right\} \subset B C(S)$ is defined by $\chi_{n}(x)=\psi_{n}\left(x \quad a_{n} e_{3}\right), x \in S$. From Assumption Al it follows that $K \psi_{n}\left(x \quad a_{n} e_{3}\right)=K \chi_{n}(x)$. Thus

$$
\left\|\chi_{n} \quad K \chi_{n}\right\|_{\infty}=\left\|\psi_{n} \quad K \psi_{n}\right\|_{\infty} \rightarrow 0
$$

as $n \rightarrow \infty$.

Since $\left\{\chi_{\mathrm{n}}\right\}$ is bounded in $B C(S)$, by Lemma 2.3 we can choose a subsequence $\left\{\chi_{n_{m}}\right\}$ and $\chi \in B C(S)$ such that $K \chi_{n_{m}} \rightarrow \chi$. From (14) it follows that $K \chi_{n_{m}} \rightarrow \chi$ and then, from Lemma 2.4, that $K \chi_{n_{m}} \rightarrow \mathrm{K} \chi$. Thus $\chi=K \chi$. Since $\|\chi\|_{\infty} \geq$ $\inf _{x \in \widetilde{\Gamma}}\left|\chi_{\mathrm{n}}(x)\right| \geq \frac{1}{2}$ and $I-K$ is injective we have a contradiction.

In the next two theorems we commence an examination of conditions for ( $I-$ $K) B C(S)=B C(S)$ and of the convergence of $\phi_{\mathrm{a}}$, the solution of the integral equation (9) on $S_{\mathrm{a}}$, to $\phi$ as $a \rightarrow \infty$

THEOREM 2.6. Suppose that $I-K$ is injective and that, for some $A>0$ and all $a \geq A,\left(I \quad K_{a}\right)^{1}$ exists and is a bounded operator on $B C(S a)$, with $C:=$ $\sup a \geq A \|\left(I \quad K_{a}\right)^{1 \|}<\infty$. Then $(I-K)^{-1}$ exists as an operator on $B C(S)$ with $\|(I \quad K) \quad 1\| \leq C$ Moreover, if $g \in B C(S),\left\{a_{n}\right\} \subset \mathrm{R}^{+}$and $a_{n} \rightarrow \infty$, then $(I-$ $\left.K_{a_{n}}\right){ }^{1} E_{a n} g \rightarrow\left(\begin{array}{ll}I & K\end{array}\right){ }^{1} g$. 
Proof. Let $g \in B C(S)$ and define the sequence $\left\{\phi_{n}\right\}$, where $\phi_{n} \in B C\left(S_{a_{n}}\right)$ for each $n$, by $\phi_{n}=\left(I-K_{a_{n}}\right){ }^{1} E_{a_{n}} g$. Then

$$
\phi_{n}=K_{a_{n}} \phi_{n}+E_{a_{n}} g .
$$

Since $\left\{\phi_{n}\right\}$ is a bounded sequence, by Lemma 2.3 there exists a subsequence $\left\{\phi_{n_{m}}\right\}$ and $\phi \in B C(S)$ such that

$$
K_{a_{n_{m}}} \phi_{n_{m}}+E_{a_{n_{m}}} g \rightarrow \phi .
$$

Thus, from (15), $\phi_{n_{m}} \rightarrow \phi$ Hence, from Lemma 2.4, $K_{a_{n_{m}}} \phi_{n_{m}} \rightarrow K \phi$, and thus $K_{a_{n_{m}}} \phi_{n_{m}}+E_{a_{n_{m}}} g \rightarrow K \phi+g$. Thus $\phi=K \phi+g$ and so $g \in(I-K) B C(S)$ and we have shown that $I-K$ is surjective, so that $(I-K)^{-1}$ exists as an operator on $B C(S)$.

By the same argument, given $g \in B C(S)$, every subsequence of $(I-K)^{-1} E_{a_{n}} g$ has a subsequence converging to $\left(I-K_{a_{n}}\right)^{-1} g$ Thus, by Remark 2.5,

$$
\left(I-K_{a_{n}}\right)^{-1} E_{a_{n}} g \rightarrow(I-K)^{-1} g
$$

for every $g \in B C(S)$. From this and Remark 2.3 it follows that $\left\|(I-K)^{-1}\right\| \leq C$.

THEOREM 2.7. Suppose that the conditions of the previous theorem are satisfied. Define $\phi_{n}:=\left(I-K_{a_{n}}\right)^{-1} E_{a_{n}} g$ and $\phi:=(I-K)^{-1} g$. If $\phi(x) \rightarrow 0$ as $x \rightarrow \infty$ then $\left\|\phi \quad R_{a_{n}} \phi_{n}\right\|_{\infty} \rightarrow 0$ as $n \rightarrow \infty$

Proof. By definition,

$$
\phi_{n} \quad K_{a_{n}} \phi_{n}=E_{a_{n}} g
$$

and $\phi \quad K \phi=g$, so that

$$
E_{a_{n}} \phi \quad K_{a_{n}} \phi_{n}=E_{a_{n}} g .
$$

From (16) and (17) it follows that

$$
\phi_{n}-E_{a_{n}} \phi=\left(I-K_{a_{n}}\right)^{-1} x n,
$$

Where $x_{n}=K_{a_{n}} E_{a_{n}} \phi-E_{a_{n}} K \phi$, so that

$$
\left\|\phi_{n}-E_{a_{n}} \phi\right\| \infty \leq C\left\|X_{n}\right\| \infty .
$$

For each $n$, let $A_{n}:=\left(a_{n}-1\right) / 2$. By Assumptions $A 1$ and $A 2$, and from the definition of $\Phi$,

$$
\begin{aligned}
& \sup _{x \in S}\left|\bar{a}_{n}\right| E_{a_{n}} K(x)\left|\leq \sup _{x \in \bar{S} a_{n} \mid \bar{S} \text { an }-1}\right| K \phi(x) \mid
\end{aligned}
$$

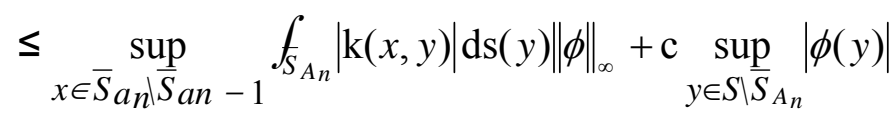

$$
\begin{aligned}
& \leq \sup _{\mathrm{t} \geq \mathrm{A}_{\mathrm{n}}} \Phi(\mathrm{t})\|\phi\|_{\infty}+\mathrm{c} \sup _{\mathrm{y} \in \mathrm{S} \backslash \widetilde{\mathrm{S} A n}}\|\phi(\mathrm{y})\| .
\end{aligned}
$$

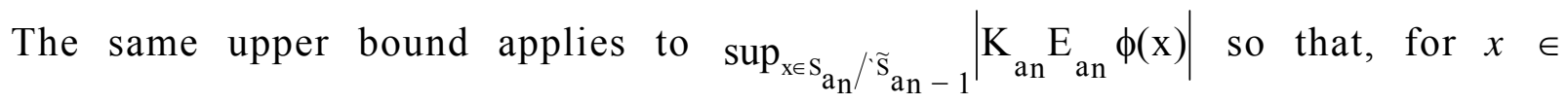
$\mathrm{S}_{\mathrm{a}_{\mathrm{n}}} \backslash \overline{\mathrm{S}}_{\mathrm{an}-1}$

$$
\left|\mathrm{X}_{\mathrm{n}}(\mathrm{x})\right| \leq 2 \sup _{\mathrm{t} \geq \mathrm{A}_{\mathrm{n}}} \Phi(\mathrm{t})\|\phi\|_{\infty}+2 \mathrm{c} \sup _{\mathrm{y} \in \mathrm{S} \backslash \widetilde{\mathrm{S}} \mathrm{An}}|\phi(\mathrm{y})|
$$


For, $\mathrm{x} \in \widetilde{\mathrm{S}}_{\mathrm{a}_{\mathrm{n}-1}}$,

$$
X_{n}(x)=\int_{S_{a_{n}} \backslash \bar{S}_{a_{n-1}}} k(x, y)\left(E_{a_{n}} \phi\right)(y) d s(y) \quad \int_{S \backslash \bar{S}_{a_{n-1}}} K(x, y) \phi(y) d s(y),
$$

So that

$$
\left|X_{n}(x)\right| \leq 2 c \sup _{y \in S \backslash \bar{S}_{\text {an }}-1}|\phi(y)| \leq 2 \mathrm{c} \sup _{y \in S \backslash \widetilde{S}_{A_{n}}}|\phi(y)|
$$

Thus

$$
\left\|\mathrm{X}_{\mathrm{n}}\right\|_{\infty} \leq 2 \sup _{\mathrm{t} \geq \mathrm{A}_{\mathrm{n}}} \Phi(\mathrm{t})\|\phi\|_{\infty}+2 \mathrm{c} \sup _{\mathrm{y} \in \mathrm{S} / \overline{\mathrm{S}}_{\mathrm{An}}}|\phi(\mathrm{y})| .
$$

so that, by Lemma 2.1, $\left\|X_{n}\right\|_{\infty} \rightarrow 0$ |and, by (18), $\left\|\phi_{n}-E_{a_{n}} \phi\right\|_{\infty} \rightarrow 0$ as $n \rightarrow \infty$

Now,

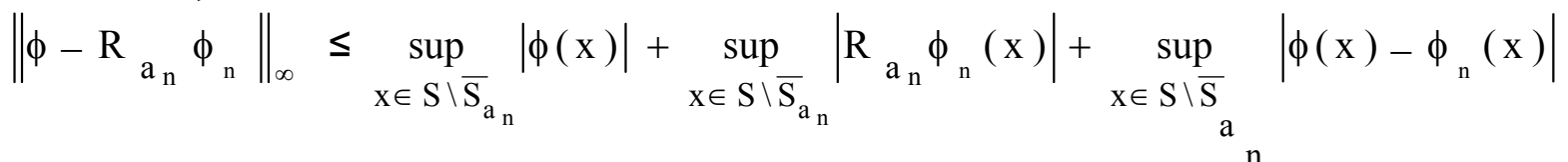

$$
\begin{aligned}
& \leq 2 \sup _{x \in S \mid \bar{S}_{a_{n}}}|\phi(x)|+2 \sup _{x \in \bar{S}_{a_{n}}}\left|\phi(x)-\phi_{n}(x)\right| \\
& \leq 3 \sup _{x \in S \backslash \widetilde{S}_{a_{n-1}}}|\phi(x)|+2\left\|+\phi_{n}-E_{a_{n}} \phi\right\|_{\infty}
\end{aligned}
$$

S ince $\left|\phi(x)-E_{a_{n}} \phi(x)\right| \leq|\phi(x)|, x \in \overline{\mathrm{S}} \backslash \overline{\mathrm{S}}_{a_{n-1}}$ Thus $\left\|\phi-R_{a_{n}} \phi_{n}\right\|_{\infty} \rightarrow 0$ a s $n \rightarrow \infty$

The next theorem is a criterion for the existence of a solution to equation (9) for all sufficiently large $a \geq A$ and the uniform stability of the approximate inverse operators.

THEOREM 2.8. If $I-K, I-K_{+}$, and $I-K$ are injective, then $\left(I-K_{a}\right)^{-1}$ exists and is a bounded operator on $B C\left(S_{a}\right)$ for all sufficiently large $a \geq A$ and $\mathrm{C}:=\sup _{\mathrm{a} \geq \mathrm{A}}\left\|\left(\mathrm{I}-\mathrm{K}_{\mathrm{a}}\right)^{-1}\right\|<\infty$.

Proof. Note that if $\left(I-K_{a}\right)^{-1}$ exists (i.e. $I-K_{a}$ is injective) then, since $K_{a}$ is compact, $\left(\mathrm{I}-\mathrm{K}_{\mathrm{a}}\right\}^{-1}$ is a bounded operator on $\mathrm{BC}\left(\mathrm{S}_{\mathrm{a}}\right\}$ by the Fredholm Alternative.

Suppose now that the theorem is false. Then there exist sequences $\left\{a_{n}\right\} \subset R^{+}$ and $\left\{\psi_{n}\right\}$ such that $a_{n} \rightarrow \infty, \psi_{n} B C\left(S_{a_{n}}\right)$ and $\left\|\psi_{n}\right\|_{\infty}=1$ for each $n$, and

$$
\left\|\psi_{\mathrm{n}}-\mathrm{K}_{\mathrm{a}_{\mathrm{n}}} \psi_{\mathrm{n}}\right\|_{\infty} \rightarrow 0 \text {. }
$$

Since $\left\|\psi_{\mathrm{n}}\right\|_{\infty}=1$ we can find a sequence $\left\{x^{(n)}\right\}$ such that $x^{(n)}=\left(x_{1}^{(n)}, x_{2}^{(n)}, x_{3}^{(n)}\right) \in$ $S_{a_{n}}$ for each n and $\left|\psi_{n}\left(x^{(n)}\right)\right| \geq \frac{1}{2}$.

There are two cases to consider: $(a) a_{n} \quad\left|x_{3}^{(n)}\right| \rightarrow \infty$ as $\mathrm{n} \rightarrow \infty ;(b)\left\{a_{n}\left|x_{3}^{(n)}\right|\right\}$ is bounded.

CASE (a). For each $\mathrm{n}$, define $T_{n}:=S_{a_{n}}-x_{3}^{(n)}$ and $X_{n} \in B C\left(T_{n}\right)$ by

Then, by Assumption Al, it is easy to see that

$$
x_{n}(x)=\psi_{n}\left(x+x_{3}^{(n)} e_{3}\right), \quad x \in T_{n} .
$$

$$
K_{a_{n}} \psi_{n}\left(x+x_{3}^{(n)} e_{3}\right)=K_{T_{n}} X_{n}(x), \quad x \in T_{n} .
$$


CASE (b). We choose subsequences $\left\{a_{n_{m}}\right\}$ and $\left\{x_{3}^{(n m)}\right\}$ such that either $\left\{a_{n_{m}-}\right.$ $\left.x_{3}^{(n m)}\right\}$ is bounded or $\left\{a_{n_{m}}+x_{3}^{(n m)}\right\}$ is bounded. Define $T_{m}:=S_{a_{n_{m}}} \operatorname{T} a_{n_{m}}$ in the case $\left\{a_{n_{m}} \mp x_{3}^{(n m)}\right\}$ bounded, and $x_{m}$, for each $\mathrm{m}$, by

$$
x_{m}(x)=\psi_{n_{m}}\left(x \pm a_{n_{m}} e_{3}, x \in T_{m} .\right.
$$

Then

$$
K_{a_{n_{m}}} \psi_{n_{m}}\left(x \pm a_{n_{m}}\right)=K_{T_{m}} X_{m}(x), x \in T_{m} .
$$

Clearly, in both cases (a) and (b), $\left\|X_{m}\right\|_{\infty}=1$ for each $m$ and, from equations (20) and (21) in Case (a), (22) and (23) in Case (b),

$$
\left\|X_{m}-K_{T_{m}} X_{m}\right\|_{\infty}=\left\|\psi_{n_{m}}-K_{a_{n_{m}}} \psi_{n_{m}}\right\|_{\infty} \rightarrow 0
$$

as $m \rightarrow \infty$. Note also that $T_{m} \rightarrow T$, where $T=S$ (Case (a)) or $T=S \pm$ in the case. $\left\{a_{n_{m}} \mp x_{3}^{\left(n_{m}\right)}\right\}$ bounded (Case (b)), and that, for some $A>0$,

$$
\sup _{x \in T_{m},|x| \leq A}\left|X_{m}(x)\right| \geq \frac{1}{2}
$$

for all $m$.

Applying Lemma 2.3, $\left\{K_{T_{m}} X_{m}\right\}$ has a subsequence $\left\{K_{T_{m}} X_{m j}\right\}$ such that $K_{T_{m j}} X_{m j} \rightarrow$ $X \in B C(T)$. From (24) it follows that $X_{m j} \rightarrow X$ and then, from Lemma 2.4, that $K_{T_{m j}} X_{m j} \rightarrow K_{T X}$. Thus $X=K_{T X}$ but, by (25), $\|X\|_{\infty} \geq \frac{1}{2}$. This is a contradiction since $T=S, S_{+}$, or $S$-, so that $I-K_{T}$ is injective.

Combining these results, Theorems 2.6-2.8, we have immediately the following corollaries, conditions for the solvability of equation (1) and of (9) and for the convergence of $\phi_{a}$ to $\phi$.

COROLLARY 2.9. If $I-K, I-K_{+}$, and $I-K$ are injective, then $(I-K)^{-1}$ exists and is a bounded operator on $B C(S)$, so that equation (1) has a unique solution $\phi$ for all $g \in B C(S)$.

COROLLARY 2.10. If $I-K, I-K_{+}$, and $I-K_{-}$are injective, then equation (9) has a solution, $\phi_{\mathrm{a}}$ for all sufficiently large $a \geq A$. Moreover, $\phi_{\mathrm{a}} \rightarrow \phi$ the solution of equation (1), as a $\rightarrow \infty$. Further, if $\phi(x) \rightarrow 0$ as $x \rightarrow \infty$, then $\left\|R_{a} \phi_{a}-\phi\right\| \infty \rightarrow 0$.

3. Numerical Solution of the Truncated Equations. In this section we extend the arguments of Section 2 to investigate the convergence of a simple piecewise constant finite element collocation method applied to the truncated equation (9) in the limit $a \rightarrow \infty$ and $h \rightarrow 0$, where $h$ is the diameter of the largest element of the mesh.

For $T \in \sum$ bounded we call $\Pi=\left\{\gamma^{(1)}, \ldots . . \gamma^{(N)}\right\}$ a mesh on $\mathrm{T}$ if, for each $i$, $\gamma^{(i)} \subset T$ is open in $T, \gamma^{(i)} \cap \gamma^{(j)}=\theta, i \neq j$, and $\bigcup_{i=1}^{N} \overline{\gamma^{(i)}}=\mathrm{T}$ For a mesh II let $h(\Pi)$ denote the diameter of the largest element of $\Pi$.

Given a sequence $\left\{T_{n}: n \in N\right\} \subset \Sigma$ (each $T_{n}$ bounded) and a mesh $\Pi_{n}=$ $\left\{\gamma_{n}^{(1)}, \ldots, \gamma_{n}^{\left(N_{n}\right)}\right\}$ on $T_{n}$, for each $n$, one can construct a sequence of piecewise constant interpolation operators $\left\{P_{n}: n \in N\right\}$, where $P_{n}: B C\left(T_{n}\right) P_{n}: B C\left(T_{n}\right) \rightarrow L_{\infty}\left(T_{n}\right)$ defined by

$$
P_{n} \psi(x)=\psi\left(x_{n}^{(i)}\right), x \in \gamma_{n}^{(i)}, i=1, \ldots . N_{n},
$$

where $x_{n}^{(i)}, i=1, \ldots N_{n}, n=1,2, \ldots .$, is a given interpolation point in $\gamma_{n}^{(i)}$. 
REMARK 3.1. If $T_{n} \rightarrow T \in \sum, \psi_{n} \in B C\left(T_{n}\right)$ for each $n, \psi_{n} \rightarrow \psi \in B C(T)$, and $h_{n}:=h\left(\Pi_{n}\right) \rightarrow 0$, then, for each $A>0$ and all sufficiently large $n$,

$$
\begin{aligned}
& \sup _{x \in T,|\mathrm{x}| \leq A}\left|P_{n} \psi_{n}(x) \quad \psi(x)\right| \leq \sup _{\mathrm{x} \in \mathrm{T},|\mathrm{x}| \leq \mathrm{A}+\mathrm{h}_{\mathrm{n}}}\left|\psi_{n}(x) \quad \psi(x)\right| \\
& \sup _{x, x^{\prime} \in T,|x|,\left|x^{\prime}\right| \leq A+h_{n},}\left|x-x^{\prime}\right| \leq h_{n}\left|\psi(x)-\psi\left(x^{\prime}\right)\right| \rightarrow 0
\end{aligned}
$$

as $n \rightarrow \infty$, so that $P_{n} \psi_{n} \rightarrow \psi$.

We have the following lemma, analogous to Lemma 2.4, a continuity property of the operator sequence $\left\{K^{(n)}\right\}$, where $K^{(n)}: B C\left(T_{n}\right) \rightarrow B C\left(T_{n}\right)$ is denned by

$$
K^{(n)}=K_{T_{n}} P_{n} \text {. }
$$

LEMMA 3.1. Suppose that $\left\{T_{n}\right\} \subset \sum, T \in \sum, \psi_{n} \in B C\left(T_{n}\right)$ for each $n$, $\psi \in B C(T), \quad\left\{P_{n}\right\}$ is the sequence of piecewise interpolation operators given by (26), $\psi_{n} \rightarrow \psi$ and $h_{n} \rightarrow 0$. Then $K^{(n)} \psi_{n} \rightarrow K_{T \psi}$.

Proof. Apply Lemma 2.4 and Remark 3.1 to the sequence $\left\{P_{n} \psi_{n}\right\}$.

Throughout the remainder of the section we suppose that the following additional assumption is satisfied:

A5. $\left\{P_{n}\right\}$ and $\left\{K^{(n)}\right\}$ are denned by (26) and (27), with $T_{n}=S_{a_{n}}$ for $n \in \mathrm{N}$, and $\left\{a_{n}\right\} \subset[2, \infty)$. Also, $a_{n} \rightarrow \infty, h_{n}:=h\left(\prod_{n}\right) \rightarrow 0$, as $n \rightarrow \infty$.

Suppose that Assumption A5 is satisfied and, for $n \in \mathrm{N}$, define $\tilde{\phi}^{(\mathrm{n})}$, a piecewise constant finite element collocation method approximation to $\phi_{a_{n}}$, by

$$
P_{n} \tilde{\phi}^{(n)}=P_{n} E_{a_{n}} g+P_{n} K_{a_{n}} \tilde{\phi}^{(n)}, \quad \tilde{\phi}^{(n)} \in P_{n} B C\left(S_{a_{n}}\right)
$$

Sloan [12] proposed using the iterated collocation solution rather than the collocation method approximation. The iterated collocation solution, $\phi^{(n)} \in B C\left(S_{a_{n}}\right)$, is obtained by applying one Neumann iteration to $\tilde{\phi}^{(n)}$ : thus

$$
\phi^{(n)}: \mathrm{E}_{\mathrm{a}_{\mathrm{n}}} \mathrm{g}+\mathrm{K}_{\mathrm{a}_{\mathrm{n}}} \tilde{\phi}^{(\mathrm{n})} \text {. }
$$

Note that $\tilde{\phi}^{(n)}=P_{n} \phi^{(n)}$, so that $\phi^{(n)}$ and $\tilde{\phi}^{(n)}$ agree at the collocation points, and $\phi^{(\mathrm{n})}$ satisfies equation (10). From the results of Sloan [12] it can be expected that $\phi^{(\mathrm{n})}$ will approximate $\phi_{a_{n}}$ more accurately than $\tilde{\phi}^{(n)}$ and we concentrate on proving the convergence of $\phi_{a_{n}}$ in the remainder of this section. Note that, since $\tilde{\phi}^{(n)}=P_{n} \phi^{(n)}$,

$$
\tilde{\phi}^{(n)} \rightarrow \phi \quad \text { if } \phi^{(n)} \rightarrow \phi,
$$

by Remark 3.1.

We now present results on the stability and convergence to $\phi$ of the iterated collocation solution, $\phi^{(\mathrm{n})}$. Our results are discrete versions of Theorems 2.6-2.10 of the last section.

THEOREM 3.2. Suppose that $I$ - $K$ is injective and that, for all sufficiently large $n \geq N,\left(I-K^{(n)}\right)^{-1}$ exists and is a bounded operator on $B C\left(S_{a_{n}}\right)$ and $\sup _{n \geq N}$

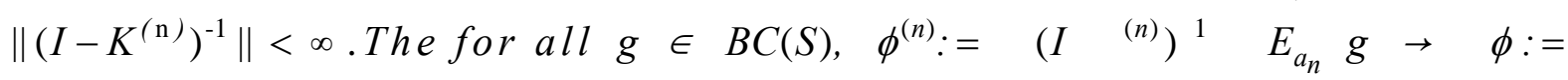
$(\mathrm{I}-\mathrm{K})^{-1} \mathrm{~g}$ as $n \rightarrow \infty$. If also $\phi(x) \rightarrow \infty$, then $\left\|\phi-\mathrm{R}_{\mathrm{a}_{\mathrm{n}}} \phi^{(\mathrm{n})}\right\|_{\infty} \rightarrow 0$.

Proof. Let $\mathrm{g} \in B C(S)$ and define the sequence $\left\{\phi^{(n)}\right\}$ as above. Then

$$
\phi^{(\mathrm{n})}=\mathrm{K}^{(\mathrm{n})} \phi^{(\mathrm{n})}+\mathrm{E}_{\mathrm{a}_{\mathrm{n}}} \mathrm{P}_{\mathrm{n}} \phi^{(\mathrm{n})}+\mathrm{E}_{\mathrm{a}_{\mathrm{n}}} \mathrm{g} .
$$


Since $\sup _{n \geq N}\left\|P_{n} \phi^{(n)}\right\|=\sup _{n \geq N}\left\|\phi^{(n)}\right\|<\infty$, by Lemma 2.3 there exists a subsequence $\left\{\phi^{\left(n_{m}\right)}\right\}$ and $\phi \in B C(S)$ such that

$$
K^{\left(n_{m}\right)} \phi^{\left(n_{m}\right)}+E_{a_{n_{m}}} g \rightarrow \phi
$$

Thus, from (28), $\phi^{\left(n_{m}\right)} \rightarrow \phi$. The proof that $\phi^{(n)} \rightarrow \phi$ now follows that of Theorem 2.6, utilising Lemma 3.1 in place of Lemma 2.4. Similarly, the proof that $\| \phi-$ $R_{a_{n}} \phi^{(n)} \|_{\infty} \rightarrow 0$ as $n \rightarrow \infty$ follows that of Theorem 2.7.

THEOREM 3.3. If $I-K, I-K_{+}$, and $I-K$ - are infective, then $\left(I-K^{(n)}\right)^{-1}$ exists and is a bounded operator on $B C\left(T_{n}\right)$ for all sufficiently large $n \geq N$ and $\sup _{n \geq N}\left\|\left(I-K^{(n)}\right)^{-1}\right\|<\infty$.

Proof. Suppose that the theorem is false. Then, arguing as in the proof of Theorem 2.8, it follows that there exists a subsequence $\left\{K^{\left(n_{m}\right)}\right\}$ and a sequence $\left\{\psi_{m}\right\}$ with $\psi_{m} \in B C\left(S_{a_{n_{m}}}\right)$ and $\left\|\psi_{m}\right\|_{\infty}=1, \quad m \in N$, such that

$$
\left\|\psi_{m} \quad K^{\left(n_{m}\right)} \psi_{m}\right\|_{\infty}=\left\|\psi_{m} \quad K_{a_{n_{m}}} P_{n_{m}} \psi_{m}\right\|_{\infty} \rightarrow 0 .
$$

Since $\left\|\psi_{m}\right\|_{\infty}=1$, we can find a sequence $\left\{x^{(m)}\right\}$ such that $x^{(m)}=\left(x_{1}^{(m)}, x_{2}^{(m)}, x_{3}^{(m)}\right)$ $\in S_{a_{n_{m}}}, m \in \mathrm{N}$, and $\left|\psi^{(m)}\left(x^{(m)}\right)\right| \geq \frac{1}{2}$.

Now, arguing as in proof of Theorem 2.8, one can construct a sequence $\left\{T_{m}\right\} \subset$ $\sum$ such that $T_{\mathrm{m}} \rightarrow T \in\left\{S, S_{+}, S_{-}\right\}$, a sequence $\left\{\chi_{m}\right\}$, with $\chi_{m} \in B C\left(T_{m}\right\}$ and $\sup _{x \in T_{m}},|x| \leq A\left|\chi_{m}(x)\right| \geq \frac{1}{2}$ for some $A>0$ and each $m$, and a sequence of piecewise constant interpolation operators, $\left\{P_{m}^{*}\right\}$, where $P_{m}^{*}: B C\left(T_{m}\right) \rightarrow L_{\infty}\left(T_{m}\right)$ for each $m$, such that

$$
\left\|\chi_{m} \quad K_{T_{m}} P_{m}^{*} \chi_{m}\right\|_{\infty} \quad \rightarrow \quad 0, \quad h\left(\Pi_{m}^{*}\right) \rightarrow 0
$$

as $m \rightarrow \infty$, where $\prod_{m}^{*}$ is the mesh on $T_{m}$ corresponding to the operator $P_{m}^{*}$. For example, in Case (a) of the proof of Theorem $2.8\left(a_{n_{m}}\left|x_{3}^{(m)}\right| \rightarrow \infty\right.$ as $\left.m \rightarrow \infty\right)$,

$$
\begin{array}{r}
T_{m}:=S_{a_{n_{m}}} x_{3}^{(m)}, \\
\chi_{m}(x):=\psi_{m}\left(x+x_{3}^{(m)} e_{3}\right), x \in T_{m}, \\
\prod_{m}^{*}:=\prod_{m} x_{3}^{(m)},
\end{array}
$$

and, for $\psi^{*} \in B C\left(T_{m}\right)$,

$$
P_{m}^{*} \psi^{*}(x):=P_{n} \psi\left(x+x_{3}^{(m)} e_{3}\right), \quad x \in T_{m},
$$

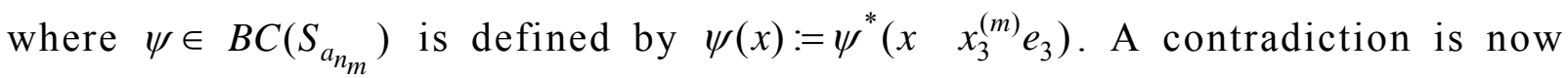
obtained as in the last paragraph of the proof of Theorem 2.8, utilising Lemma 3.1 in place of Lemma 2.4 .

Combining the two theorems, we obtain the following, our main convergence result for the iterated collocation solution.

COROLlary 3.4. If $I-K, I-K_{+}$, and $I-K$ - are injective, then, for all sufficiently large $n \geq N$, equation (10) has a solution, and $\phi^{(n)} \rightarrow \phi$, the solution of (1), as $n \rightarrow \infty$. Further, if $\phi(x) \rightarrow 0$ as $x \rightarrow \infty$, then $\left\|R_{a_{n}} \phi^{(n)} \quad \phi\right\|_{\infty} \rightarrow 0$. 
4. An Application to Acoustic Scattering. We apply the results of the previous sections to the case in which $S$ is the surface of an acoustically rigid cylinder embedded in a homogeneous fluid occupying $D$, the exterior of $S$.

For $T \in \sum$ let $n(x)$ denote the normal to $T$ at $x$, directed into the exterior of $T$, and let $D_{T}$ denote either the interior or the exterior of $T$. Following Colton and Kress [9], let $\mathfrak{R}\left(D_{T}\right)$ denote the linear space of all complex functions $\Psi \in C^{2}\left(D_{T}\right) \cap B C\left(\overline{D_{T}}\right)$ such that:

(i) the normal derivative on the boundary exists in the sense that the limit

$$
\frac{\partial u(x)}{\partial v}=\lim _{h \rightarrow 0, h>0} \nabla u(x+h v(x)) \cdot v(x), \quad x \in T,
$$

exists uniformly on compact subsets of $T$, where $v(x)=n(x)$ if $D_{T}$ is the exterior domain, $v(x)=-n(x)$ if $D_{T}$ is the interior domain;

(ii)

$$
\sup _{x \in T, 0<h<\epsilon}|\nabla u(x+h v(x)) \cdot v(x)|<\infty
$$

for some $\in>0$;

(iii)

$$
\sup _{x \in D_{T}, \operatorname{dist}(x, T) \geq \in}|\nabla u(x)|<\infty
$$

for all $\in>0$,

Note that if $u \in \mathfrak{R}\left(D_{T}\right)$ then, by (i) and (ii), $\frac{\partial u}{\partial n} \in B C(T)$.

Consider the scattering of an incident acoustic wave, bounded and continuous on $S$, and of angular frequency $\omega$ ( $e^{i \omega t}$ time dependence). This gives rise to the following boundary value problem for the space dependent part of the scattered field.

BVP1 Find $u \in \mathfrak{R}(D)$ such that

$$
\begin{aligned}
\Delta u+\kappa^{2} u & =0 \quad \text { in } \quad D, \\
\frac{\partial u}{\partial n} & =F \quad \text { on } \quad S .
\end{aligned}
$$

In equation (33), $F \in B C(S)$ is given $\left(\mathrm{F}=-\frac{\partial \mathrm{u}^{\mathrm{i}}}{\partial \mathrm{n}}\right.$, where $u^{i}$ is the incident field) as is $\kappa \in C$. We will assume throughout that $\operatorname{Im} \kappa>0$, so that the medium of propagation is lossy. The results we obtain apply only partially to the case $\operatorname{Im} \kappa>0$.

This boundary value problem can be reformulated as a boundary integral equation. Define $G(x, y)$ by equation (4). For $R>0$ let $B_{R}:=\left\{x \in R^{3}:|x|<R\right\}$. For $h>0$ sufficiently small, let $S^{h}:=\{x+h n(x): x \in S\}$, so that $S^{h}$ is parallel to and distance $h$ from $S$ and of class $C^{l}$ [9, p.37], and let $D^{h}$ be the region exterior to $S^{h}$. Applying Green's representation theorem [9, p. 68] in $D^{h} \cap B_{R}$, letting $R \rightarrow \infty$, noting that $G(x, y)$ decays exponentially as $|x-y| \rightarrow \infty$, and that $u$, are bounded in $\overline{D^{h}}$ by (31), we obtain

$$
u(\mathrm{x})=\int_{s^{h}} u(y) \frac{\partial G(x, y)}{\partial n(y)}-\frac{\partial u(y)}{\partial n} G(x, y) \quad d s(y), \quad x \in D^{h} .
$$

Letting $h \rightarrow 0$, and utilising equations (29), (30), and (33), we see that

$$
u(x)=U(x)+\int_{S} \frac{\partial G(x, y)}{\partial n(y)} u(y) d s(y), \quad x \in D,
$$


Where

$$
U(x):=-\int_{S} F(y) G(x, y) d s(y), \quad x \in \bar{D} .
$$

Equations (34) and (35) represent $u$ in $D$ as the combination of a single- and a doublelayer acoustic surface potential. Prom standard properties of these potentials [9] it follows that $U \in B C(\overline{D)}$ (in fact $U \in \mathfrak{R}(D)$ ) and that the double-layer in (34) is continuous up to the boundary $S$, its limiting value given by [9, Thm 2.13]. Thus, letting $x$ in (34) approach $S$, we find that $\phi:=u \mid s \in B C(S)$ satisfies the following boundary integral equation:

$$
\phi(x)=g(x)+2 \int_{S} \frac{\partial(x, y)}{\partial n(y)} \phi(y) d s(y), \quad x \in S,
$$

where $g:=2 U \mid s \in B C(S)$. This equation is of the form (1) with $k(x, y)$ given by (3). Using a mixture of elementary and standard arguments (e.g. [9, p. 50]) it can be seen that $k$, given by (3), satisfies Assumptions A1-A4 (for details see Peplow [11]).

In order to apply the results of the previous sections we need also to consider the same integral equation but with $S$ replaced by $S_{+}, S_{-}$or $S_{a}$, for some $a>0$. We will show uniqueness of solution of equation (36), and of the same equation with $S$ replaced by $S_{+}$or $S_{-}$, by using the following uniqueness result for corresponding boundary value problems. Note that this result, in particular, shows that BVP1 has at most one solution.

THEOREM 4.1. Suppose that $T \in \sum, D_{T}$ is either the interior or exterior of $T$, and $v \in \mathfrak{R}\left(D_{T}\right)$ satisfies

$$
\Delta v+\kappa^{2} v=0 \text { in } D_{T}
$$

and either

$$
v=0
$$

or

$$
\frac{\partial v}{\partial n}=0
$$

on $T$. Then $v=0$.

Proof. For $h>0$, define $T^{h}=\{x+v(x) h: x \in T\}$. Then $T^{h}$ is the parallel surface distance $h$ from $T$ lying in $D_{T}$ Let $D_{T}^{h}$ be the region exterior (interior) to $T^{h}$ if $D_{T}$ is exterior (interior) to $T$. For $\in>0$, define $F_{\in} \in C^{\infty}\left(\mathrm{R}^{3}\right)$ by $F_{\in}(x)=$ $\exp \left(-\in \sqrt{1+|x|^{2}}\right)$.

Applying Green's first theorem to $\bar{v}$ and $v F_{\in}$ in the region $B_{R} \cap D_{T}^{h}$, utilising equation (37), then letting $R \rightarrow \infty$, we obtain

$$
\begin{aligned}
\int_{D_{T}^{h}} F_{\in}\left\{\bar{\kappa}^{2}|v|^{2}+\left.\nabla v\right|^{2}\right\} & d x+\int_{\partial D_{T}^{h}} F_{\epsilon} v \frac{\partial \bar{v}}{\partial v} d s \\
= & -\int_{D_{T}^{h}} v \nabla F_{\epsilon} \cdot \nabla \bar{v} d x
\end{aligned}
$$

Letting $h \rightarrow 0$ and utilising (38) or (39) we find that the integral over $\partial D_{T}^{h}$ vanishes and equation (40) holds with $D_{T}^{h}$ replaced by $D_{T}$. Multiplying this equation by $\kappa$, 
and taking the imaginary part, we obtain

$$
\operatorname{Im} \kappa\left(P_{\epsilon}^{2}+q_{\epsilon}^{2}\right)=\operatorname{Im}\left\{\kappa \int_{D_{T}} v \nabla F_{\epsilon} \cdot \nabla \bar{v} \quad d x\right\}
$$

where $p_{\in}:=\left\{\int_{D_{T}} F_{\epsilon}|\kappa|^{2}|v|^{2} d x\right\}^{\frac{1}{2}}, q_{\in}:=\left\{\left.\int_{D_{T}} F_{\in} \nabla v\right|^{2} d x\right\}^{\frac{1}{2}}$. Noting that $\left|\nabla F_{\in}\right| \leq \in F_{\in}$ and applying the Cauchy-Schwarz inequality, we obtain

$$
\operatorname{Im} \kappa\left(P_{\epsilon}^{2}+q_{\in}^{2}\right) \leq \in p_{\in} q_{\epsilon} \text {. }
$$

It follows that $\operatorname{Im} \kappa p_{\epsilon} \leq \in q_{\epsilon}$ and $\operatorname{Im} \kappa \mathrm{q}_{\epsilon} \leq \in \mathrm{p}_{\epsilon}$ so that $(\operatorname{Im} \kappa)^{2} \mathrm{p}_{\epsilon} \mathrm{q}_{\epsilon}$. Thus, for $\in<\operatorname{Im} \kappa, p_{\in}=0$. Thus $v=0$ in $D_{T}$.

Using the above result, the following theorem shows that both equation (1) and equation (9), with $k$ defined by (3), have at most one solution. Note that in this next theorem and through to the end of this section $K_{T}$ is defined by equation (6) with $k$ defined by (3). We give a fairly brief version of the proof of Theorem 4.2 - the result is in any case standard in the case $T$ bounded [9] — for further detail see Peplow [11].

THEOREM 4.2. For all $T \in \sum, I-K_{T}$ is injective on $B C(T)$.

Proof, Suppose that $T \in \sum, \psi \in B C(T)$ and $\psi=K_{T \psi}$. From [9, Theorem 2.30] (which applies immediately if $T$ is bounded, with some additional argument, if $T=S, S_{+}$or $\left.S_{-}\right), K_{T}$ maps $B C(T)$ onto the Hölder space $C^{0, \alpha}(T)$, and $C^{0, \alpha}(T)$ onto $C^{1, \alpha}(T)$. It follows that $\psi \in C^{1, \alpha}(T)$.

Let $D_{T}$ denote the exterior of $T$ and define

$$
\begin{aligned}
v(x) & :=\int_{T} \frac{\partial G(x, y)}{\partial n(y)} \psi(y) d s(y), \quad x \in D_{T}, \\
w(x) & :=\int_{T} \frac{\partial G(x, y)}{\partial n(y)} \psi(y) d s(y), \quad x \in \mathrm{R}^{3} \backslash \overline{D_{T}} .
\end{aligned}
$$

Clearly

$$
\begin{gathered}
\Delta v+\kappa^{2} v=0 \quad \text { in } \quad D_{T}, \\
\Delta w+\kappa^{2} w=0 \quad \text { in } \quad R^{3} \backslash \overline{D_{T}} .
\end{gathered}
$$

From [9, Theorem 2.13 and 2.23], and noting that $\int_{T} \frac{\partial G(x, y)}{\partial n(y)} \psi(y) d s(y)=\frac{1}{2} \psi(x)$, $x \in T\left(\right.$ since $\left.\psi=K_{T} \psi\right)$, it follows that $v \in \quad\left(D_{T}\right), \quad w \in \quad\left(R^{3} \backslash \overline{D_{T}}\right)$ and

$$
\begin{aligned}
& v=\psi, \\
& w=0,
\end{aligned}
$$

and

$$
\frac{\partial v}{\partial n}=\frac{\partial w}{\partial n}
$$

on T. Noting (44) and (46) and applying Theorem 4.1, it follows that $\omega=0$ in $\mathrm{R}^{3} \backslash \overline{D_{T}}$, so that $\frac{\partial w}{\partial n}=0$ on $T$. Then, noting (43) and (47) and applying Theorem 4.1, it follows that $v=0$ in $\overline{D_{T}}$ and, from (45), that $\psi=0$. We have shown that $I-K_{T}$ is injective.

Having shown that the homogeneous version of (1), with $S$ replaced by $T$, has no non-trivial solution, for all $T \in \sum$, we can now obtain existence of solution to 
equation (1) and equation (9), convergence of $\phi_{a} \rightarrow \phi$ as $a \rightarrow \infty$, and convergence of the numerical approximation $\phi^{(n)}$, denned by equation (10). Applying Corollary 2.9 and Theorem 4.2 we have immediately

COROLLARY 4.3. The integral equation (36) has precisely one solution.

From Theorem 2.8, Corollary 2.10 and Theorem 4.2 we have

COROLlary 4.4. Equation (9), with $k$ defined by (3), has a solution, $\phi_{a}$, for every $a \geq 2$. For some $A>0$, $\sup _{a \geq A}\left\|\left(I K_{a}\right)^{1}\right\|<\infty$. Further, $\phi_{a} \rightarrow \phi$ as $a \rightarrow \infty$, and, if $\phi(x) \rightarrow 0$ as $x \rightarrow \infty$, then $\left\|R_{a} \phi_{a} \quad \phi\right\|_{\infty} \rightarrow 0$ as $a \rightarrow \infty$.

Finally, from Theorem 3.3, Corollary 3.4 and Theorem 4.2 we have

COROLLARY 4.5. Suppose that Assumption A5 is satisfied. Then equation (10), with $k$ defined by (3), has a solution, $\phi^{(\mathrm{n})}$ for all sufficiently large $n \geq N$, and $\sup _{n \geq N}\left\|\left(I K^{(n)}\right)^{1}\right\|<\infty$. Further, $\phi^{(n)} \rightarrow \phi$ as $n \rightarrow \infty$, and, if $\phi(x) \rightarrow 0$ as $x \rightarrow \infty$, then $\left\|R_{a_{n}} \phi^{(n)} \phi\right\|_{\infty} \rightarrow 0$ as $n \rightarrow \infty$.

We remark that the proof of Theorem 4.2, which shows, inter alia, that, if $\phi$ satisfies equation (36) with $g=0$, then $v$ satisfies BVP1 with $F=0$, can be extended (see Peplow [11]) to show that BVP1 and the integral equation (36) (with $g:=2 U \mid s$ ) are equivalent, in that, if $\phi$ satisfies (36), then $u$, defined on $S$ by $u \mid \mathrm{s}=\phi$, and in $D$ by (34), satisfies BVP1. (We have already shown conversely that if $u$ satisfies BVP1 then $u \mid$ s satisfies (36).) Thus Corollary 4.3 also establishes, by integral equation methods, unique existence of solution of BVP1.

\section{REFERENCES}

[1] P. M. ANSELONE, Collectively Compact Operator Approximation Theory and Applications to Integral Equations, Prentice Hall, Englewood Cliffs, NJ 1971.

[2] P. M. ANSELONE AND I. H. SLOAN, Integral equations on the half-line, J. Integral Eq., 9 (1985), pp. 3-23.

[3] _ Numerical solutions of integral equations on the half-line. II. The Wiener-Hopf case, J. Integral Eq. Appl., 1 (1988), pp. 203-225.

[4] _ Spectral approximations for Wiener-Hopf operators, J. Integral Eq. Appl., 2(1990),PP. 237-261.

[5] K. E. ATKINSON, The numerical solution of integral equations on the half-line, SIAM J. Numer. Anal., 6 (1969), pp. 375-397.

[6] C. A. BREBBIA, J. C. F. TELLES, AND L. C. Wrobel, Boundary Element Techniques, Springer-Verlag, Berlin, 1984.

[7] R. C. BUCK, Bounded continuous functions on a, locally compact space, Michigan Math. J., 5 (1958), pp. 95-104.

[8] S. N. CHANDLER-WILDE, Some uniform stability and convergence results for integral equations on the real line and projection methods for their solution, IMA J. Numer. Anal., 13 (1993), pp. 509-535.

[9] D. COLTON AND R. KRESS, Integral Equation Methods in Scattering Theory, Wiley-Interscience Publication, New York, 1983.

[10] - Inverse Acoustic and Electromagnetic Scattering Theory, Springer-Verlag, Berlin, 1992.

[11] A. T. PEPLOW, Boundary Integral Equation Methods in Outdoor Sound Propagation, PhD thesis (in preparation), University of Bradford, UK.

[12] I. H. SLOAN, Superconvergence and the Galerkin Method for Integral Equations of the Second Kind, in Numerical Treatment of Integral Equations, C.T.H. Baker and G.F. Miller, eds., Academic Press, London, 1982.

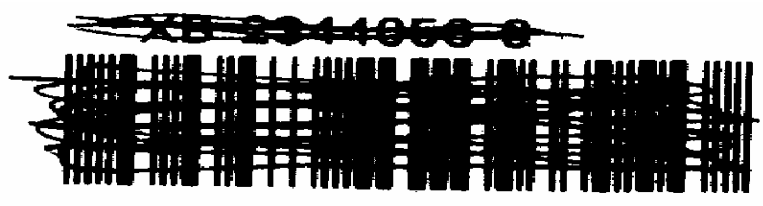

